\title{
Research on the Forecast Model of Total Viable Count on Bacon Based on Hyper spectral Imaging Technique
}

\author{
Junhua Zhao, Peiyuan Guo, Pan Xu, Shuo Liu and Kuncheng Yang
}

School of Computer and Information Engineering, Beijing Technology and Business University, Beijing 100048, China

\begin{abstract}
The total viable count (TVC) in bacon overweight can cause serious damage to human health .In order to find a rapid and nondestructive method of TVC, hyper spectral imaging technique was applied to quantitatively analysis of TVC on bacon .Comprehensively comparing the pretreatment method of multiple scattering, derivative method and so on ,finally the multiple scattering for pretreatment was used. And the interval optimization method of least squares model was set up to predict, and get a good prediction results.The correlation coefficient of the calibration and predictions respectively was 0.808 and 0.808 , interactive authentication root mean square error was 0.115 and 0.198 respectively. Therefore, hyper spectral imaging technique combining iPLS can be used for the rapid detection of TVC on bacon.
\end{abstract}

Key words: bacon; total viable count; hyper spectral imaging; interval optimization least-squares

\section{Introduction}

Chinese bacon is an important heritage of world food culture, however, research shows: due to the environmental health conditions of the slaughter process is not up to standard, bacon will produce a large number of microbial residues which is not conductive to long-term preservation of bacon, and harmful metabolites will be increased accordingly that will result in a decline in quality of bacon. So there is an urgent need for a new real-time, fast ,accurate detection of bacon quality.

The total viable count (TVC) is a very important microbiological indexes to evaluate the quality of the bacon health .Most of the traditional detection methods are based on the method of microscope ,the method of plate counting, etc. ${ }^{[5]}$.But most of them in detection method are low efficient, have long time-consuming cycle, and have the disadvantage of breaking meat and so on .

As a new detection technology, hyper spectral imaging technology ${ }^{[6]}$ set of optoelectronics, computer science and other fields of cutting-edge technology in a body and conventional spectroscopy and imaging techniques also be combined together. The superiority of hyper spectral imaging technology ${ }^{[7]}$ is reflected in the following aspects: Have a high spectral resolution, the resolution can be got NM level, and number of bands up to hundreds, so multiple narrow spectrum obtained can be approximated as a continuous; Atlas unity--hyper spectral image contains both spectral information inside the substances, but also image information outside the substances make it more accurate.

This experiment takes bacon as the research object and detects the total number of bacteria on the surface of the bacon. In $900 \mathrm{~nm}-1700 \mathrm{~nm}$ wavelength range of hyper spectral imaging system, acquire image and spectral information of the sample, determine the best modeling characteristic wavelengths, and then establish the prediction model based on interval partial least squares to analyze and compare .Through the above steps, we can achieve quality and safety detection about bacteria on the surface of bacon. 


\section{Material and Methods}

\subsection{Materials}

The bacon in Leshan, Sichuan was taken as the experimental object. The collected sample is divided into $4 \mathrm{~cm} \times 4 \mathrm{~cm} \times 2 \mathrm{~cm}$ (length $\times$ width $\times$ thickness), and 40 samples were collected. In order to accelerate the process of bacon corruption, expose it to the $35{ }^{\circ} \mathrm{C}$ storage space of moisture , air turbidity to be tested .Two meat samples taken randomly every $5 \mathrm{~d}$ were tested during the experiment--one piece as the calibration set samples, another piece as the prediction set sample until all the samples out.

\subsection{Instrument}

Hyper spectral imaging data were acquired by GaiaSorter hyper spectral sorter systems from Beijing Zhuo Li Han Kuang Instrument Co., Ltd as shown in Figure 1.

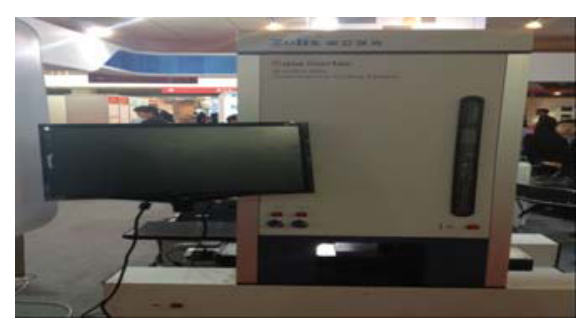

Figure 1. Spectrum analyzer system of sisuCHEMA.

\subsection{The acquisition of spectral values and detection of total bacterial count}

\subsubsection{The acquisition of hyper spectral image}

In order to overcome the influence of the image noise and dark current in the wavelengths of weak light intensity distribution, first you need to scan standard white to acquire all white calibration image Dw with the reflectivity of 1 , then cover the lid of the camera to acquire all black calibration image Dd with the reflectivity of 0 . After over a black and white correction, then the spectral image of sample Ds can be acquired. It can be based on Formula 1 to get reflectivity $\mathrm{R}$ of the interested pixel region $\mathrm{C}$ or band $\mathrm{I}$ of the relative spectral image of sample after a black and white corrected.

$$
R(c i)=\frac{D_{s}(c i)-D_{d}(c i)}{D_{w}(c i)-D_{d}(c i)}
$$
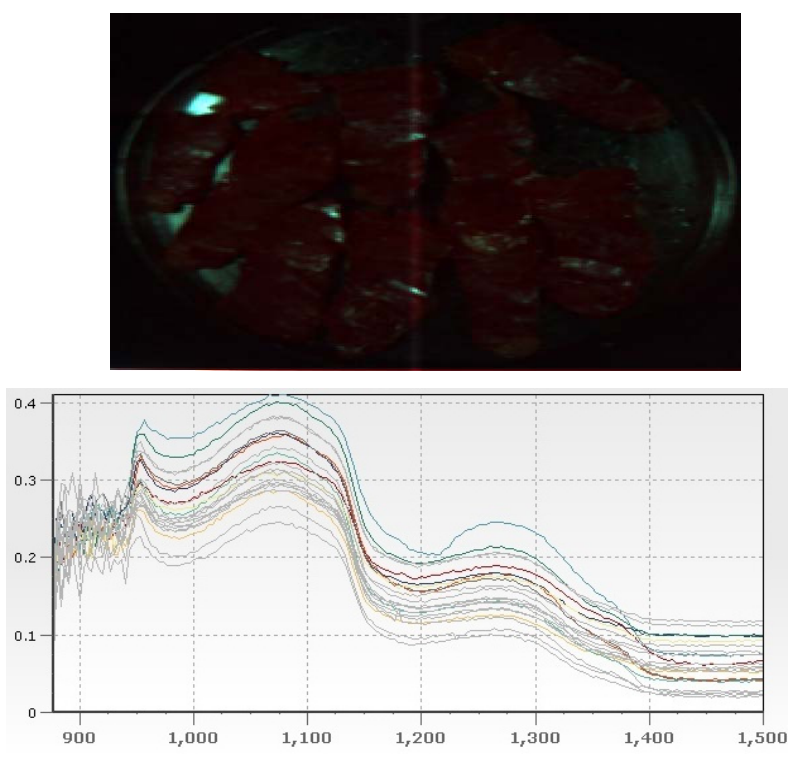

Figure 2. The original spectral on bacon.

The data analysis of hyper spectral image used ENVI software platform. To the hyper spectral images of sample corrected, select a region of interest on the surface and calculate the average reflectance spectroscopy .According to this method sequentially obtain a full-band spectral reflectance curves of the original of sample which are shown in Figure 2.

\subsubsection{Determination microbes on the surface of} the sample

The microbial detection experiments were carried out immediately after obtaining the spectral images of all the samples. Total number of colonies: In accordance with the GB/10278-1997" the total number of colonies of Chinese bacon" to determinate. Using the method of plate counting, select $2-3$ sample dilutions of suitable dilution , and take $1 \mathrm{~mL}$ empty diluent and then place it in a sterile petri dish. Cool the $15 \mathrm{~mL}$ medium of plate counting, flip it until the agar solidified, and then cultivate it for $48 \mathrm{~h}$ in the condition of $36^{\circ} \mathrm{C}$. The total numbers of colonies gathered are shown in Figure 3, and the data was used as the standard reference data.

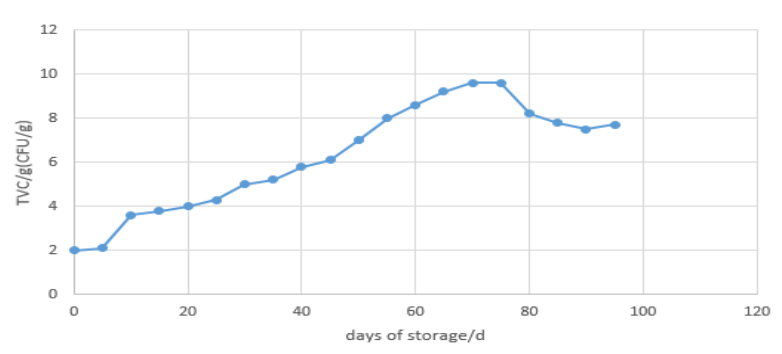

Figure 3. Bacon bacterial colonies. 


\section{Results and Analysis}

\subsection{Spectral data preprocessing}

In this study, the original spectra were pretreated by using differential pretreatment, vector normalization, multiple scatter correction and the combination of the methods, etc. Finally make a model of the bacon, and use correlation coefficient $\mathrm{R}$ and root mean square error of cross- validation (RMSEVC) as 2 evaluation index of the model. While $\mathrm{R}$-value is closer to 1 , and RMSEVC value is smaller, the performance of the model is better. It can be drawn that within $900-1700 \mathrm{~nm}$ wavelength range, the best spectral preprocessing method for modeling is multivariate scatter correction (MSC). This is mainly because MSC not only can effectively eliminate the scattering influence produced by the sample particles spotty and uneven distribution, but also can correct the error of optical path length of hyper spectral scanning sample. The model adopting MSC and standardized preprocessing method is optimal in which $\mathrm{R}$ is 0.934 , the RMSECV is 0.186 .

Using the above preprocessing method to preprocess hyper spectral data of sample of calibration set of the bacon collected, the spectrum are shown in Figure 4.

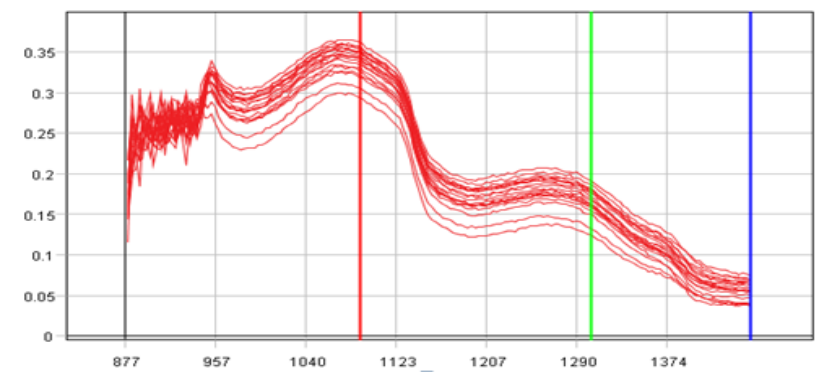

Figure 4. The pretreatment of spectrum.

\subsection{Establishment of forecasting model of partial least squares for interval optimization(iPLS)}

The method of partial least square is to eliminate redundant information and noise produced during the process of modeling, so decompose and change not only the matrix $\mathrm{X}$ of high spectral, but also the matrix $\mathrm{Y}$ of bacterial content of samples of the bacon to be detected. So at the same time, the effect of matrix $Y$ on $\mathrm{X}$ is considered. The method of PLS makes the direct relationship between main components of $X$ and $Y$. In addition, it can reduce the space of data dimension letting the high-dimensional data into low dimensional, so as to solve the nonlinear problems.

Due to the high spectrum carries the information of the whole structure of the material, in the process of analyzing and modeling of the spectral data, the phenomenon of the loss of target information in the spectra was avoided by using full spectrum data to establish the model. But when the band range of the spectrum is very large, it is easy to cause the information redundancy, the model complexity, and the reduction of accuracy of the model if still make model using the data of full spectrum. In the study, in order to avoid the above problems, the forecasting model is established by using the method of regression analysis of the interval partial least squares.

Use the method of regression analysis of interval partial least squares to establish prediction model .Full spectrum is divided equally into $10,11,12,13 \ldots 20$ interval, and because the value of $\mathrm{n}$ does not have the corresponding theoretical formula, the classification is on the basis of experience. PLS regression analysis was carried out in every sub interval divided, and the accuracy of model was evaluated by RMSECV. The smaller the value of the RMSECV, the better prediction ability of the model. In the case the high spectrum is divided into different numbers of sub regions. When the spectrum is divided equally into 12 regions, the number of the factor of principal components is determined for 10 , and the analysis model of the total number of bacteria in bacon is optimal. At this point, the best range of local model is 8 . The scatter plot of the actual and predicted value of the TVC on bacon surface got by using the hyper spectra processed as the input of partial least squares model are shown in Figure 5. The correlation coefficient $r$ was 0.808 and RMSECV was 0.115 . 


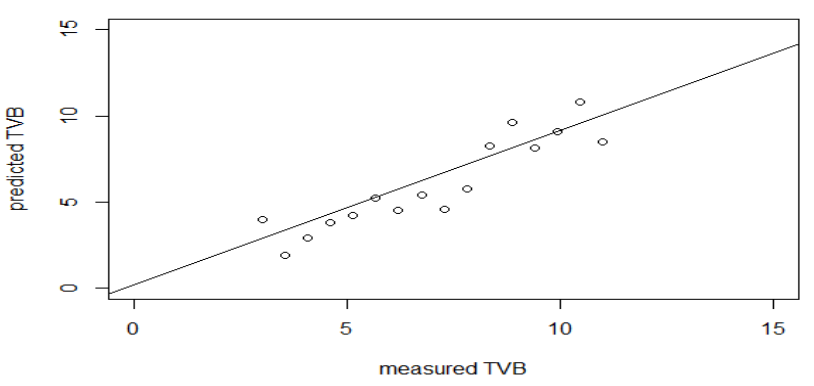

Figure 5. The scatter plot of the actual and predicted value of the TVC on bacon surface.

Enter the sample of bacon of validation set into the model of analysis prediction of the total number of bacteria in the bacon created by the model of regression analysis of interval partial least squares, compare the result of outputs of model and the true value obtained through the national standard method of measurement, and the results are shown in Figure 6. Blue represents the real value, and red represents the predicted value.

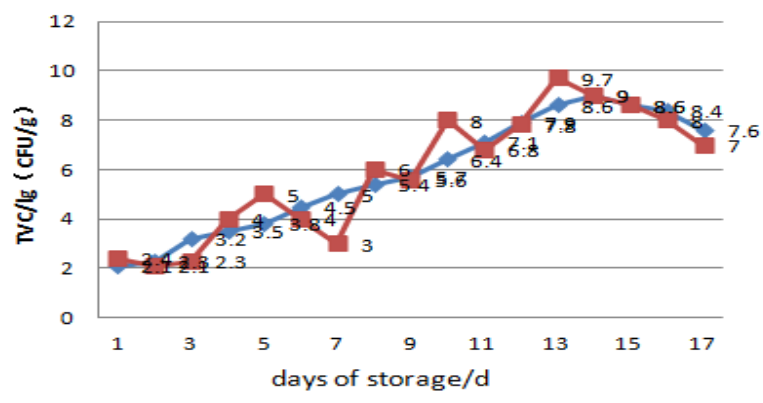

Figure 6. The comparison of the actual and predicted value of the TVC on bacon surface.

From the predicted results of the model, we can learn that correlation coefficient of test results by the detection model of content of the total number of bacteria on the surface of bacon established by modeling method of interval partial least squares and actual results is 0.798 , and the error of root mean square of cross validation is 0.198 .

\section{Conclusion}

In order to avoid the long time and the destruction of the sample of the traditional detection of the bacteria, the experiment adopted nondestructive testing by hyper spectra to the content of bacterial on the surface of bacon, and achieved the expected effect. In this paper, create a predictive model by using the hyper spectral technology combined with MSC + standardized calibration and interval partial least squares. But later need to increase the number of the samples of prediction set and calibration set, and hope to get better results.

\section{References}

1. H.H. Wang, D.Y. Liu, et al, Journal of meat research.The situation and development trend of bacon industry present in China. J, 27 (9) : 36 to 40(2013)

2. W. Z. Sun, C. Cui, et al, Journal of food science and technology. The research on influential factors of acid on Cantonese sausage storage . J.(2007)

3. L. G. Zhou, L. Xiao, et al, Journal of meat research, Near infrared spectroscopy rapidly detect bacon acid value and peroxide value. J, 26 (3) : 30-33(2012)

4. L. L. Zhao, L. D. Zhang, et al, Spectroscopy and spectral analysis. The research on bacon quality by near infrared spectrum . J, 27 (1) : 46-49(2007)

5. N. N. Li, G.H. Zhou, et al, Journal of food industry science and technology. The residues of pyrene in bacon by chromatographyfluorescence. J, 34 (1) : 319-323(2013)

6. L. L. Tao, X.J. Yang, et al, Spectroscopy and spectral analysis. The research progress on the chemical composition by near infrared spectrum in poultry. J, 33 (11) : 3002-3009(2013)

7. J. H. Wu, Beijing: China agricultural university. The forecast of fresh beef quality by hyper spectral. D. (2010)

8. K. W. Liu, F. Cheng, et al, Spectroscopy and spectral analysis. The detection on fat ,protein and moisture content in fresh pork by visible/near-infrared spectrum.

$\mathrm{J}$, 29(1):102-105(2009)

9. [9] R. D. Dong, X. C Wang, et al, Spectroscopy and spectral analysis. The detection nondestructively and rapidly on the content of phosphate in by infrared spectrum. J, 
33(6): 1542-1546(2013)

10. [10] X. B. Zhou, X. L. Zhang, et al, Journal of agricultural engineering. The detection on the content of cucumber leaf chlorophyll based on hyper spectral image. J, 30(13):169-175(2014)

11. [11] Y. Y. Li, L. L. Zhang, Y. K. Pen, et al, Sensing for Agriculture and Food Quality and Safety . Hyper spectral Imaging Technique for Determination of Pork Freshness Attributes., $1-9(2011)$

12. [12] M. Kamruzzaman, et al, Analytica Chimica Acta . Prediction of Some Quality Attributes of Lamb Meat Using Near-Infrared Hyper spectral Imaging and Multivariate Analysis, 714:57-67(2012)

13. [13] J. Zhao, Y. K. Pen, et al, Journal of agricultural engineering. The evaluation on beef tender degree Based on hyper spectral image texture feature. J, 31(7):279-286(2015)

14. [14] D. Barbin, et al, Analytica Chimica Acta. Predicting Quality and Sensory Attributes of Pork Using Near-Infrared Hyper spectral Imaging, 719:30-43(2012) 